Conclusions Similar to other studies, condom use was more likely to be reported by persons most at risk; however, even among those at increased risk, fewer than half used condoms during most recent sexual intercourse.

\section{P2-S3.05 ASSOCIATION OF STI-RELATED STIGMA AND SHAME TO STI TESTING AND PARTNER NOTIFICATION AMONG YOUNG BLACK MEN IN SAN FRANCISCO}

doi:10.1136/sextrans-2011-050108.324

${ }^{1} S$ Lippman, ${ }^{1} \mathrm{~J}$ Morris, 'M Lightfoot, ${ }^{2} \mathrm{~S}$ Philip. 'University of California, San Francisco, USA; ${ }^{2}$ San Francisco Department of Public Health, STD Prevention and Control Services, San Francisco, USA

Background Young minority men in the US bear a disproportionate burden of STI, but comprise one of the population groups least likely to access services. STI service utilisation is not only constrained by access to quality care but also potentially impacted by socio-cultural factors, including STI-related stigma and shame, which can undermine efforts to promote testing and treatment. STI-related stigma and shame may also provide a disincentive for young men to participate in partner notification programs, including partnerdelivered therapy. We hypothesised that young men who perceive increasing levels of social stigma related to STI would be less likely to seek out STI-related services or notify their sexual partners about STI. Methods Between June and July, 2010, 108 African American young men (15-24 years) responded to a brief, self-administered intercept survey on a hand-held device. Recruitment was conducted on the street and in residential areas of a low income urban neighbourhood with elevated STI rates. The survey included socio-demographic questions, an 11 item scale measuring STI-related stigma and shame, and questions regarding STI testing history, preferences for notifying partners, and interest in partner delivered therapy. The association between stigma and shame scores and STI testing and partner notification preferences was evaluated with multivariate logistic regression, adjusting for age and education.

Results The median (range) STI-stigma score was 12 (5-25) and the shame score was 15 (6-30); higher scores indicate more stigma or shame. Most participants had ever been tested for STI (73\%), indicated willingness to personally notify their main partners $(72 \%)$ or other partners (66\%), and said they would deliver STI therapy to a partner (68\%). Increasing STI-related stigma was significantly associated with a history of STI testing, such that every SD increase in stigma score was associated with $50 \%$ decreased odds of having been tested (OR: 0.5, 95\% CI 0.3 to 1.0). Participants with higher levels of stigma and shame were also significantly less likely to be willing to personally notify their partners of STI or to deliver therapy. Conclusions STI-related stigma and shame, common in this population, could undermine STI testing, treatment, and partner notification programs. Efforts to expand access to care should be accompanied by efforts to change socio-cultural attitudes and norms around STI testing and treatment.

\section{P2-S3.06 EXPLORING SEXUAL IDENTITY DEVELOPMENT OF AFRICAN AMERICAN MALE COLLEGE STUDENTS AGE 18-25 AT A HISTORICALLY BLACK COLLEGE AND UNIVERSITY}

doi:10.1136/sextrans-2011-050108.325

${ }^{1} \mathrm{~S}$ Randolph, ${ }^{2} \mathrm{C}$ Golin, ${ }^{2} \mathrm{D}$ Matthews. ${ }^{1}$ North Carolina Agricultural and Technical State University, Greensboro, USA; ${ }^{2}$ The University of North Carolina, Chapel Hill, USA

Among the estimated 56000 new yearly infections of HIV in the USA, $51 \%$ are among African Americans. This demonstrates a disproportionate burden of HIV infection as African Americans make up approximately $12 \%$ of the population. One group warranting attention in North Carolina has been African American male college students. Between 2000 and 2003, 11\% of new HIV infections among men ages 18-30 were enrolled in college at the time of their diagnosis, with $87 \%$ of those college students being African American. Another examination of HIV transmission among men ages $18-30$ in North Carolina revealed that $15 \%$ of the men reported sexual contact with both men and women in the year prior to their diagnosis, and that these individuals were more likely than men who exclusively have sex with men to be African American and enrolled in college. Sexual identity is a complex and multidimensional construct, many factors of which have yet to be sufficiently explored in the context of the sexual transmission in the HIV epidemic. This is particularly true for heterosexual men and men who have sex with both men and women, as the work that has concerned sexual identity has often ignored these two groups. This neglect may stem from the fact that sexual identity development has often been inappropriately conflated to claiming a minority sexual orientation. As a part of an attempt to more fully understand the role that sexual identity may play in the lives of African American men, we interviewed African American male college students within a historically Black college and university (HBCU) in North Carolina. Our aim was to address a gap in the literature by exploring what shapes sexual identity and its development among African American men. This could potentially lead to future research that could explain sexual behaviour within the context of the HIV epidemic for this population. Interviews were used to assess experiences, attitudes, and beliefs about sexual identity development and sexual activity held by African American male college students. A total of 31 African American male students took part in this investigation that occurred at a HBCU in the central Piedmont region of North Carolina, located in one of the largest cities within the state. Researchers developed interview questions based on The Measure of Sexual Identity Exploration and commitment (MoSIEC) survey instrument. Results from this qualitative exploratory study revealed that the ideas and beliefs about sexuality and sexual identity for African American college males are heavily impacted by their peers and their environment. Males that had influence from an older sibling, cousin, or father about sex reported less sexual partners. Majority of the males reported that it is more acceptable for men to have multiple sex partners, but it is unacceptable for a woman to do the same.

\section{P2-S3.07 RESPONDING TO PROBLEMS OF SEXUAL VIOLENCE AND VULNERABILITY TO HIV/AIDS WITHIN CRISIS AND UNSTABLE SITUATIONS: A CASE STUDY OF KENYA'S POST-ELECTION VIOLENCE}

doi:10.1136/sextrans-2011-050108.326

${ }^{1} \mathrm{C}$ Musyoka, ${ }^{2} \mathrm{~A}$ Mwendwa, ${ }^{3} \mathrm{~N}$ Kyalo. ${ }^{1}$ Walden University/The Centre for Integrated Community Development and Outreach, CICDOT Seattle, USA; ${ }^{2}$ Moi University Teaching and Referral Hospital, Eldoret, Kenya; ${ }^{3}$ University of Nairobi, Nairobi, Kenya

Introduction and Background Sexual violence and the risk of HIV transmission are clearly linked through multiple pathways underpinned by gender inequalities and poverty like increased genital injury, anal rape, multiple perpetrators and ejaculation all common in sexual violence. It is important therefore to understand and recognise these links between sexual violence and HIV/AIDS as the basis for developing strategies to address the complex interactions at individual, communal and institutional levels.

Objectives Based on the Kenya's post-election violence of 2008, this study aimed to capture and understand the complex and multiple factors that interact to influence vulnerability to HIV and AIDS. Its holistic framework proposed a macro level model for understanding the risks factors for STIs, and HIV/AIDS transmission within the context of sexual violence. Its main objective was to explore and 